Bayero Journal of Pure and Applied Sciences, 10(1): 1 - 6

ISSN 2006 - 6996

\title{
MALARIA VECTORS RESISTANCE TO COMMONLY USED INSECTICIDES IN THE CONTROL OF MALARIA IN BICHI, NORTHERN NIGERIA
}

\author{
Habibu, U. Abdu ${ }^{2 *}$, Andrew, J. Spiers ${ }^{1}$, Hapca, Simona ${ }^{1}$, Mukhtar, M. Dauda ${ }^{3}$ and Yusuf, \\ Y. Deeni ${ }^{1}$ \\ ${ }^{1}$ School of Science, Engineering and Technology, Abertay University, Dundee, UK \\ ${ }^{2}$ School of Science Education, Federal College of Education (Technical) Bichi, Kano, Nigeria \\ ${ }^{3}$ Microbiology Department, Bayero University Kano, Nigeria \\ ${ }^{*}$ Correspondence author: habibubichiusman@yahoo.com, Mobile, +2348036924792
}

\section{ABSTRACT}

This study was carried out in 2015 to assess the level of resistance of sibling species of Anopheles gambiae complex the principal malaria vector from Bichi in Kano state to three classes of insecticides; (DDT, Permethrin and Bendiocarb) approved by World Health Organization (WHO) for vector control with the aim of obtaining baseline susceptibility and resistance data by evaluating the potency of brands of insecticides used against the mosquito vector in the area where the disease is prevalent. An. gambiae larvae collected from ecologically contrasting breeding sites were reared to adults in the laboratory. Adults from the F1 progeny were assayed for resistance using the WHO adult insecticide susceptibility bioassay protocol. The mosquitoes were exposed to DDT, permethrin and bendiocarb belonging to 3 classes of insecticides. The individual sibling species were identified molecularly and the molecular resistance mechanisms characterized by SINE and kdr PCR methods respectively. The An. gambiae populations tested revealed high level of resistance to DDT and permethrin but less resistant to bendiocarb. Analysis of the genetic composition of the vector population revealed predominance of An. coluzzii (> 77\%). L1014F and L1014S kdr mutations correlated to insecticide resistance phenotype expression. This study indicates differential distribution of the resistant genotype of Anopheles malaria vector between ecologically different habitats in the area. This insecticide resistance represents a threat to vector control tool and should be monitored. The information obtained could be useful in decision and planning making for vector control programs in the region.

Key words-Anopheles coluzzii, Anopheles gambiae, Insecticides, Malaria control, resistance, Susceptibility

\section{INTRODUCTION}

Anopheles gambiae Giles is one of the most important malaria vectors in Africa. Malaria kills more people than HIV/AIDS or any other killer disease. Malaria is endemic throughout Nigeria accounting for $25 \%$ of infant mortality (Federal Ministry of Health, 2005). Malaria impedes on economic development not only by causing premature death but also through lost/diminished productivity, enormous medical cost and population growth. Malaria vector control programs in Africa rely heavily on the use of pesticides for insecticide-treated nets (ITNs)/long-lasting insecticide-treated nets (LLINs) and for indoor residual spraying (IRS) (Kabula et al. 2014). The two primary methods of vector control; Indoor Residual Spraying (IRS) and the distribution of Long-Lasting InsecticideTreated Nets (LLINs) have been demonstrated to reduce the number of malaria cases when properly used against insecticide susceptible mosquito populations (Wondji et al. 2012). The use of both strategies has substantially increased over the years since 2000 in many malaria endemic countries with increased donor funding to achieve Roll Back Malaria targets aimed at the elimination of malaria. The overall ownership of the nets has increased steadily from $8 \%$ in 2008 to $42 \%$ in 2010 , so also the proportion vulnerable group sleeping under net increased from $6 \%$ to $29 \%$ in the same period (Kolade et al. 2013). The development and rapid spread of insecticides resistance to major malaria vectors across the African countries has posed a threat to effectiveness of these different mosquito control measures (Kolade et al., 2013). This resistance is mainly associated with either reduced target site sensitivity arising from a single point mutation in the sodium channel gene, or increased metabolic detoxification of insecticides. The knockdown resistance (kdr) characterized by changing either a Leucine residue to a Phenylalanine; West African mutation (L1014F) or a Leucine to Serine; East African mutation (L1014S) have being identified in An. gambiae and confers knockdown resistance (kdr) to DDT and pyrethroid insecticides. 
The metabolic resistance involves increased detoxification through enzymatic activities of the large enzyme families' esterases, glutathione s-transferases and monooxygenases.

Information on the susceptibility of principal malaria vectors to common insecticide used in public health and the underlying mechanism are crucial. This information will adequately inform control programs of the most suitable insecticide to use and facilitate the design of appropriate resistance management strategies. However, recently (Ibrahim et al. 2014; and Habibu et al, 2017) have both reported high frequency of $\mathrm{kdr}$ L1014F associated with pyrethroid resistance in Auyo. In this study, we provided additional information on the differential distribution of the genotypic resistance on the agricultural and residential vector populations, established their species and resistance status to Permethrin, DDT and Bendiocarb and detected the kdr-mutations resistance markers.

\section{MATERIALS AND METHODS}

\section{Study sites}

The study area is located within the Sudan savannah zone of West Africa about 840 kilometres from the edge of the Sahara desert (Ibrahim et al., 2014). Kano sees on average about $690 \mathrm{~mm}$ (27.2 in) of precipitation per year. The study localities in Bichi are characterized by high insecticides usage for agricultural or personal protection (1) Bichi residential $\left(12^{0} 13^{\prime} \mathrm{N}, 8^{0} 15^{\prime} \mathrm{E}\right)$, an urban area with high population and a major commercial centre outside Kano metropolis, (2) Bichi agricultural $\left(12^{0} 7^{\prime} \mathrm{N}, 8^{0} 14^{\prime} \mathrm{E}\right)$, an area of intensive agriculture throughout the year. Farmers in Bichi agricultural sites use a wide range of pesticides and herbicides to protect their crops. These pesticides marketed under different trade names belong to all the chemical classes including organophosphates, organochlorine, pyrethroids and carbamates.

Mosquito larval collection, processing, and rearing

Larval collections were carried out at the peak of the rainy season in 2015. Larvae and pupae of An. gambiae s.l. were collected from natural breeding sites using scoops from the two sampling sites. Larvae were transported to the insectary at Bayero University Kano. Larvae and pupae were maintained under standard insectary condition $25-28 \quad{ }^{\circ} \mathrm{C}$ and $\sim 70-80 \%$ humidity (Das, et al., 2007) supplied with baker's yeast daily. The larvae were reared in light plastic containers covered with fine mesh mosquito nets and reared in the same water from which they were sampled to maintain the impact of the environmental xenobiotics present in the breeding habitat. The adults that emerged were transferred into cages from the breeding containers.

\section{Insecticide susceptibility tests}

Mosquitoes were assessed using World Health Organization (1998) discriminating dosages with three insecticides: $0.75 \%$ Permethrin, $0.1 \%$ Bendiocarb or $4 \%$ DDT. The numbers of knockdown mosquitoes for each insecticide were recorded at every 10 minutes during the $1 \mathrm{~h}$ exposure period. After exposure, mosquitoes were kept in observatory/recovery tube and supplied with a $10 \%$ sugar solution and allowed a $24 \mathrm{~h}$ recovery period. Mosquitoes exposed to untreated filter papers served as Controls. The mortality was recorded after $24 \mathrm{~h}$, and the susceptibility status of the population graded according to WHO recommended protocol (WHO, 2013c). Dead and surviving mosquitoes from assessments were used for molecular analysis.

Mosquito species identification and PCR detection of knock-down resistance (kdr) alleles in DNA extracted from a proportion of the dead and surviving mosquito specimens from insecticide treatments were subjected to the SINE PCR (Santolamazza et al., 2008) for species and molecular form identification and also assayed for the presence of knockdown resistance (kdr) to detect the West African (L1014F) and East African (L1014S) kdr alleles based on TaqMan assay described by (Bass et al., 2007) using a Max3000P Real-Time PCR (QPCR) system (Stratagene).

Data Analysis

SPSS 22 statistical software was used to analyze the relationships. Coefficients of correlation were calculated between the frequency of different mutations, and the corresponding mortality rates between the three sampling sites.

\section{RESULTS AND DISCUSSION}

The results of the insecticides bioassays performed on adults An. gambiae s.l. are shown in Figure1. Using the WHO (2013c) definitions of resistance the two populations from the Sudan savannah (Bichi) in northern Nigeria revealed high resistance to DDT and Permethrin with low resistance to Bendiocarb. 


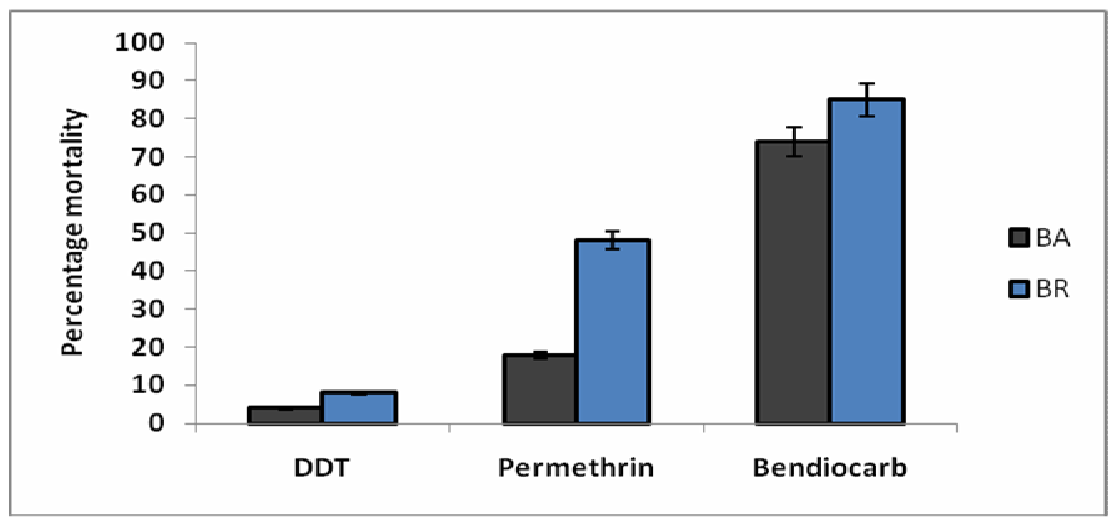

Figure 1 Mortality rate of $A n$. gambiae s.l. $24 \mathrm{~h}$ post exposure. Mosquitoes were exposed to $4 \%$ DDT, $0.75 \%$ Permethrinand $0.1 \%$ Bendiocarb in WHO susceptibility test at: Bichi agricultural (BA) and Bichi residential (BR) sites.

Table 1 Species and molecular form distribution of An. gambiae s.l. from the two different sampling sites (in Bichi northern Nigeria) using SINE based assay

\begin{tabular}{|c|c|c|c|c|c|c|c|c|}
\hline \multirow[t]{3}{*}{ Site } & & $\begin{array}{l}\text { lolecula } \\
\text { m }\end{array}$ & \multicolumn{6}{|c|}{ of An. gambiae } \\
\hline & \multicolumn{2}{|c|}{ An. arabiensis } & \multicolumn{2}{|c|}{$M$} & \multicolumn{2}{|c|}{$M / S$} & \multicolumn{2}{|c|}{$\mathrm{S}$} \\
\hline & $N$ & $\%$ & $\mathrm{~N}$ & $\%$ & $\mathrm{~N}$ & $\%$ & $\mathrm{~N}$ & $\%$ \\
\hline BA & 46 & 23.0 & 150 & 75.0 & 4 & 2.0 & 0 & 0.0 \\
\hline BR & 36 & 18.0 & 163 & 81.5 & 1 & 0.5 & 0 & 0.0 \\
\hline Total & 82 & 20.5 & 313 & 78.3 & 5.0 & 1.2 & 0 & 0.0 \\
\hline
\end{tabular}

$\mathrm{BA}=$ Bichi agricultural; $\mathrm{BR}=$ Bichi residential

Table 2 Distribution of kdr-West (L1014F) mutation in An. coluzzii and An. arabiensis mosquitoes Anopheles coluzzii Anopheles arabiensis

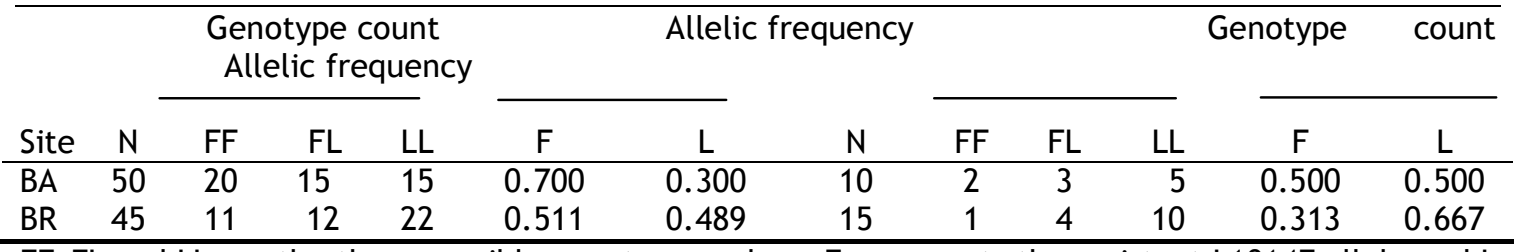

$\mathrm{FF}, \mathrm{FL}$ and $\mathrm{LL}$ are the three possible genotypes, where $\mathrm{F}$ represents the resistant $\mathrm{L} 1014 \mathrm{~F}$ allele and $\mathrm{L}$ represents the susceptible wild-type allele, $\mathrm{N}$ is number of particular species either An. coluzzii or An. arabiensis

Table 3 Distribution of kdr-East (L1014S) mutation in An. coluzzii and An. arabiensis mosquitoes

$$
\text { Anopheles coluzzii Anopheles arabiensis }
$$

\begin{tabular}{|c|c|c|c|c|c|c|c|c|c|c|c|c|}
\hline \multirow[b]{2}{*}{ Site } & \multirow[b]{2}{*}{$\mathrm{N}$} & \multicolumn{3}{|c|}{$\begin{array}{l}\text { Genotype count } \\
\text { Allelic frequency }\end{array}$} & \multicolumn{3}{|c|}{ Allelic frequency } & & & \multicolumn{2}{|r|}{ Genotype } & count \\
\hline & & SS & SL & LL & $\mathrm{S}$ & $\mathrm{L}$ & $\mathrm{N}$ & SS & SL & LL & $\mathrm{S}$ & $\mathrm{L}$ \\
\hline BA & 50 & 10 & 14 & 26 & 0.480 & 0.520 & 10 & 1 & 2 & 7 & 0.300 & 0.700 \\
\hline BR & 45 & 5 & 10 & 30 & 0.333 & 0.667 & 15 & 0 & 2 & 13 & 0.133 & 0.867 \\
\hline
\end{tabular}

SS, SL and LL are the three possible genotypes, where S represents the resistant L1014S allele and L represents the susceptible wild-type allele, $\mathrm{N}$ is number of particular species either An. coluzzii or An. arabiensis 
The mosquitoes tested from all the sampling sites showed a high resistance. Similarly, the results of the probit model showed that bendiocarb was the only insecticide that showed significant effects on the mortality of An. gambiae s.l. population exposed to these insecticides in all the study sites with $P$-values of $P<0.001$ in all the study sites. However, mosquitoes from agricultural sites (zone A) recorded higher insecticide resistance when compared to those from residential sites (zone $B)$. These high levels of resistance are probably related to extensive pesticide use in the region. Decrease in mortality rates to DDT and Permethrin was associated to a significant increase in the $\mathrm{KDT}_{50}$ observed which were higher in agricultural sites were probably due to the involvement of kdr (Bigoga et al. 2014). The higher Knockdown time observed in this study, for DDT and permethrin is consistent with the findings of (Chandre et al., 1999, Ibrahim et al., 2014, and Alhassan, et al., 2015) indicating the involvement of kdr mechanism of resistance. This is connected to the fact that the pyrethroid (permethrin) and organochlorine insecticides (DDT) shares same mechanism of action by targeting the voltage-gated sodium channel on the insects' neurons. However, the high level of resistance to Permethrin and DDT could also be associated with increased activities of the metabolic enzymes GSTs and Esterases which have been detected previously in populations of An. gambiae from Auyo resistant to these insecticides (Alhassan et al. 2015). The carbamate (Bendiocarb) on the other hand, targets the acetylcholinesterase gene (Awolola et al., 2007). DDT and Permethrin resistance pattern observed in the An. coluzzii populations are similar to previously reported cases of DDT and Permethrin resistance in the north-western, north-central and south-western Nigeria (Ibrahim et al., 2014, Alhassan et al. 2015, and Awolola et al. 2007). Selection for pyrethroid resistance in An. gambiae has been associated with the use of agricultural pesticides but not with DDT because of restricted use of DDT since it was banned (Sadasivaiah et al., 2007, Adedayo et al., 2010). But this was not the case in the present study, where some farmers confirmed the continued usage of DDT as pesticides and herbicides. This also suggests possible uncontrolled and illegal usage of DDT or other unspecified and unbranded locally made pesticides.

The massive use of pesticides in agricultural settings has been well documented as a factor contributing to the emergence of resistance in
Anopheles populations (Akogbeto et al, 2006). The sustained usage of these pesticides in the study sites may also have maintained a selection pressure for DDT resistance. The DDT and permethrin high resistance recorded in the residential areas were most likely due to increased use of household insecticides by high ITNs and IRS coverage or recurrent space spraying interventions.

The present study reveals the co-occurrence of L1014S and L1014F mutations coupled with high insecticide resistance in the two Anopheline populations belonging to agricultural and residential settings in northwest Nigeria suggesting the spreading of the L1014S mutation gene across Africa.

The high allelic frequencies recorded in the residential sites could be due to the increased use of pyrethroids for ITNs and IRS in public health as indicated in previous studies (Dykes et al. 2015; Li et al. 2015). The co-occurrence of the L1014S and L1014F mutations has already been reported in An. gambiae s.l. from both East and West Africa such as Tanzania and Nigeria (Kabula et al. 2014, and Ibrahim et al. 2014). The frequency of the L1014F mutation was positively correlated with resistance $(P=$ 0.04) thus the higher the frequency of the L1014F mutation the higher the level of resistance to DDT and permethrin. The results also show a weak relationship between the frequency of L1014F and L1014S mutations $(P=0.772)$. This study also reports the cooccurrence of East form of kdr and West form of $\mathrm{kdr}$ in An. coluzzii and An. arabiensis mosquitoes in a higher frequency than previously reported (Ibrahim et al. 2014). The low but increased frequency of the East kdr indicates that its selection in this region is recent and is increasing. No S-form was recorded from the field collections which could be explained by the previous observation made by (Coluzzii, 1984) that the $M$-form is predominant in this type of ecological setting with irrigation systems providing ideal breeding site. Increased usage of insecticides for agricultural purposes and /or widespread of LLINs and repeated use of pyrethroids in IRS in the region could explain the high frequency of the kdr mutations.

Previous studies from this region and across some African countries have reported full susceptibility to the carbamate such as bendiocarb and malathion in An. gambiae mosquitoes (Ibrahim et al., 2014) thus suggesting the use of this class of insecticide as an alternative to pyrethroids and DDT in IRS. 
Special Conference Edition, November, 2017

On the contrary, in this study An. gambiae s.l. mosquitoes tested against bendiocarb show from suspected resistance to weak resistance indicating that the An. gambiae s.l. mosquito population in this region has started to develop resistance against carbamate; however, further investigation is needed to establish the resistance status of the An. gambiae to carbamate.

Conclusion

The high insecticides resistance observed in An. gambiae s.l. populations as well as high frequency of L1014F and L1014S kdr mutation in An. coluzzii and An. arabiensis in this study particularly for pyrethroids, may compromise the efficacy of LLINs and IRS on which most African countries rely on to reduce malaria transmission. The findings from this work could help and guide the malaria control programme of Nigeria particularly in the choice of

\section{REFERENCES}

Adedayo, O.O., Olojede, J. B., Ashiegbu, C. O., Adeogun, A. O., Olubunmi, O. A. and Awolola, T. S.(2010): High level of DDT resistance in the malaria mosquito; Anopheles gambiae s.l. from rural, semi urban and urban communities in Nigeria. J. Rural Trop Public Health, 9: $114-120$

Akogbeto, M.C. Djouaka, R.F., Kinde - Gazard, D. A. (2006): Screening of pesticide residues in soil and water Samples from agricultural settings. Malar J, 5: 22

Alhassan, A. J., Sule, M. S., Dan Gambo, M. A., Yayo, A. M., Safiyanu, M. and Sulaiman, D. (2015): Detoxification Enzymes Activities in DDT and Bendiocarb resistant and susceptible malarial vector (Anopheles gambiae) breed in Auyo residential and irrigation sites, northwest Nigeria European Scientific Journal, 11: 9.

Awolola, T.S., Oduola, A., Oyewole, I.O., Obansa, J.B. Amajoh, C., Koekemoer, L. and Coetzee, M. (2007): Dynamics of knockdown pyrethroid insecticide resistance alleles in a field population of Anopheles gambiae s.s. in southwestern Nigeria. J. Vect. Borne Diseases, 44: 181-188.

Bass, C., Nikou, D., Donnelly, M. J., Willamson, M. S., Ranson, H., Ball, A., Vontas, J. and Field, L. M. (2007): Detection of knockdown resistance (kdr) mutations in Anopheles gambiae: a comparison of two new high-throughput assays with existing methods. Malar J, 6:111. insecticide.

\section{Recommendation}

It is recommended that, there should be intersectoral collaboration between pest management and vector control in agricultural and public health departments respectively towards monitoring and managing the impact of the growing market and usage of agricultural pesticides on the emergence of vector resistance and also to embark on public enlightenment on the danger and repercussions of the abuse of chemical insecticides, pesticides and herbicides both in the residential and agricultural settings towards sustainable resistance management strategies. Based on the findings of this study and also of previous studies by, Corbel et al., 2007, Akogbeto et al., 2010 and Ibrahim et al., 2014, there is a need to look into alternatives to pyrethroid insecticides to manage resistance.

Bigoga, J. D., Ndangoh, D. N., Awono-Ambene, P. H., Patchoke, S., Fondjo, E., et al. (2012): Pyrethroid resistance in Anopheles gambiae from the rubber cultivated area of Niete, South Region of Cameroon. Acta Trop 124: 210-214.

Chandre, F., Darriet, F., Manguin, S., Brengues, C., Carnevale, and P. Guillet, P. (1999): Pyrethroid cross-resistance spectrum among populations of Anopheles gambiae s.s. from Cote D'lvoire. J. Am. Mosq. Cont. Assoc. 15: 53-59

Coluzzii, M. (1984): Heterogeneities of the malaria vectorial system in tropical Africa and their significance in malaria epidemiology and control. Bull World Health Organ, 62(Suppl):107-113.

Das, S., Garver, L., Dimopoulos, G. (2007): Protocol for mosquito rearing (An. gambiae). J Vis Exp, Issue 5: 221

Dykes, C. L., Kushwah, R. S., Das, M. K., Sharma, S. N. et al. (2015): Knockdown resistance (kdr) mutations in Indian Anopheles culicifacies populations, Parasites \& Vectors, 8: 333

Federal Ministry of Health $(\mathrm{FMoH})$ : Training manual for management of malaria in Nigeria. National malaria and vector control division, Abuja Nigeria. 2005, pp. $1-86$

Habibu, U. A., Yayo, A. M., Yusuf, Y. D. (2017). Susceptibility status of Anopheles gambiae complex to Insecticides commonly used for malaria control in northern Nigeria. International journal of scientific \& Technology Research, 6 (6). 
Ibrahim, S. S., Yayo, A. M., Tukur, Z., Irving, H. and Wondji, C. S. (2014): High frequency of kdr L1014F is associated with pyrethroid resistance in Anopheles coluzzii in Sudan savannah of northern Nigeria. BMC Infectious Diseases, 14: 441.

Kabula, B., Kisinza, W., Tungu, P., Ndege, C., Batengana, B., Kollo, D., Malima, R., Kafuko, J., Mohamed, M., and Magesa, S. (2014): Co-occurrence and distribution of East(L1014S) and West (L1014F) African knock-down resistance in Anopheles gambiae sensu lato population of Tanzania. Tropical Medicine and International Health, doi. $10.1111 / \mathrm{tmi} .12248$.

Kolade, T. I., Kehinde, O. P., Oluwatobi, R. A., Adedapo, O. A. and Audu, K. O. (2013): Susceptibility of Anopheles gambiae sensu lato to permethrin, deltamethrin and bendiocarb in Ibadan city, southwest Nigeria. Current Research Journal of Biological Sciences, 5 (2): 42-48.

Li, C. X., Kaufman, P. E., Xue, R. D., Zhao, M. H., et al. (2015): Relationship between insecticide resistance and kdr mutations in the dengue vector Aedes aegypti in southern China, Parasites \& Vectors, 8: 325

Santolamazza, F., Mancini, E., Simard, F., Qi, Y., Tu, Z., Della Torre, A. (2008): Insertion polymorphisms of SINE200 retrotransposons within speciation islands of Anopheles gambiae molecular forms. Malar J, 7: 163.

Sadasivaiah, S., Tozan, Y. and Breman, J. G. (2007): Dichlorodiphenyltrichloroethane (DDT) for Indoor Residual Spraying in Africa: How can it be used for malaria control? American Journal of Tropical Medicine and Hygiene, 77: 249- 63

USAID/PMI/CDC: Nigeria FY 2012 Malaria Operational Plan. 2011,pp:46 $\mathrm{pmi}$.gov/countries/mops/fy12 /nigeria mop_fy12.pdf.

Wondji, C. S., Coleman, M., Kleinshmidt, I., Mzilahowa, T., Irving, H., Ndula, M, Rehman, A., Morgan, J., Barnes, K. G., Hemingway, J. (2012): Impact of pyrethroid resistance on operational malaria control in Malawi. Proc. Natl. Acad. Sci, 109:47 19063-70.

WHO (2013c): Test procedures for insecticide resistance monitoring in malaria vector mosquitoes. Geneva, World Health Organization. 